09,12

\title{
Ферстеровский резонансный перенос энергии с участием светлых и темных экситонов в массивах эпитаксиальных квантовых точек CdSe/ZnSe
}

\author{
(C) Т.Н. Михайлов ${ }^{1,2}$, Е.А. Европейцев ${ }^{1}$, К.Г. Беляев ${ }^{1}$, А.А. Торопов ${ }^{1}$, \\ А.В. Родина ${ }^{1}$, А.А. Головатенко ${ }^{1}$, С.В. Иванов ${ }^{1}$, Г. Позина ${ }^{3}$, Т.В. Шубина ${ }^{1}$ \\ ${ }^{1}$ Физико-технический институт им. А.Ф. Иофрфе РАН, \\ Санкт-Петербург, Россия \\ ${ }^{2}$ Санкт-Петербургский академический университет РАН, \\ Санкт-Петербург, Россия \\ 3 Линчёпингский университет, \\ Линчёпинг, Швеция \\ E-mail: tmikhaylov@mail.ru
}

\begin{abstract}
Методом спектроскопии фотолюминесценции с временным разрешением продемонстрирован ферстеровский перенос энергии между двумя массивами эпитаксиальных квантовых точек CdSe/ZnSe различных размеров, который основан на диполь-дипольном взаимодействии между основными экситонными уровнями маленьких квантовых точек и возбужденными уровнями больших точек. Показано, что зависимость эффективности переноса от ширины барьера между вставками согласуется с ферстеровским механизмом. Температурная зависимость характерных времен затухания и интенсивности фотолюминесценции свидетельствует о вовлечении в процесс переноса энергии темных экситонов.
\end{abstract}

Работа выполнена при поддержке Российского научного фонда (проект № 14-22-00107).

DOI: $10.21883 /$ FTT.2018.08.46246.03Gr

\section{1. Введение}

Эпитаксиальные квантовые точки (КT) Cd(Zn)Se/ZnSe находят широкое применение в гетероструктурах для лазеров видимого диапазона. Массивы таких КТ формируются методом молекулярно-пучковой эпитаксии (MBE) посредством осаждения дробного количества монослоев (fractional monolayer, ML) CdSe в широкозонную матрицу на основе $\mathrm{ZnSe}$ [1]. Недавно проведенные исследования [2-4] показали, что ряд спектральных и временных особенностей спектров излучения $\mathrm{Cd}(\mathrm{Zn}) \mathrm{Se} / \mathrm{ZnSe}$ КТ могут быть объяснены в рамках модели ферстеровского резонансного переноса энергии (Förster resonance energy transfer - FRET [5]). Этот нерадиационный механизм основывается на диполь-дипольном взаимодействии. Полагается, что FRET происходит между основным уровнем маленьких КТ, называемых донорами, и возбужденным уровнем больших КТ, называемых акцепторами, с последующей быстрой релаксацией энергии в нижние состояния. Схема FRET показана на рис. 1, $a$. FRET приводит к увеличению интенсивности люминесценции и сужению полосы излучения больших КТ, сопровождаемых подавлением излучения донорных точек. Отметим, что этот механизм хорошо изучен применительно к ансамблю коллоидных квантовых точек [6]. Эффективность FRET зависит от таких факторов как: расстройка от резонанса между уровнями донорных и акцепторных точек, величина дипольных моментов взаимодействующих состояний, расстояние $w$ между КТ (падает пропорционально $w^{-6}$ ). Последний фактор приводит к тому, что FRET действует в массивах КT с достаточно высокой плотностью $\left(\geq 10^{10} \mathrm{~cm}^{-2}\right)$ [4]. Теоретическое описание этого эффекта и необходимые ссылки могут быть найдены в работе [7].

Основное проявление FRET, доступное для исследования методами спектроскопии фотолюминесценции (ФЛ) с временным разрешением - увеличение скорости рекомбинации доноров в присутствии акцепторов [8]. В данной работе мы фокусируемся на изучении гетероструктур с двойными вставками массивов КТ различных размеров, где одна вставка с малыми точками рассматривается как ансамбль доноров, а другая, соответственно, как ансамбль акцепторов. При изменении толщины барьера между вставками изменяется среднее расстояние между донорами и акцепторами; таким образом, мы регулируем воздействие FRET на кинетику излучения в структуре.

Структура энергетических уровней в КТ содержит светлые AX (A - allowed) и дипольно-запрещенные темные FX (F - forbidden) экситонные состояния, которые являются, как правило, нижними по энергии в КТ (рис. $1 a$ ). Предполагается, что активационная рекомбинация темных экситонов приводит к появлению медленно затухающей компоненты в спектрах излучения. Степень вовлеченности темных экситонов в процесс переноса энергии в эпитаксиальных КТ и то, каким образом происходит этот перенос, до конца не изучены. Путем исследования структур с двумя вставками методом спектроскопии ФЛ с временным разрешением будет показано, что FRET при низких температурах 

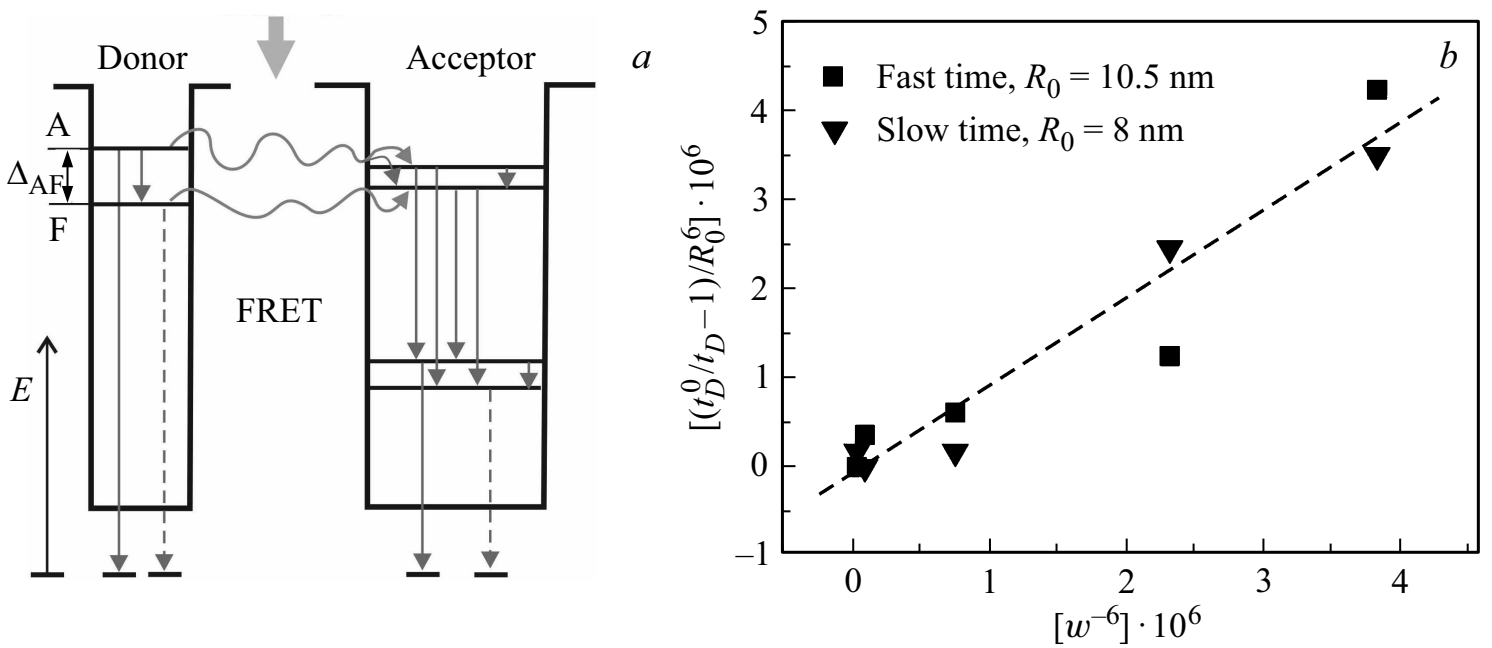

Рис. 1. (а) Схема ферстеровского резонансного переноса энергии в донорно-акцепторной системе. Релаксация и рекомбинация показана прямыми линиями. Пунктирные линии обозначают рекомбинацию из состояния темного экситона. Кривыми линиями показаны возможные варианты FRET; $\Delta_{\mathrm{AF}}$ - расщепление между состояниями темного и светлого экситонов. $(b)$ Нормированные зависимости скоростей затухания от расстояния между донорными и акцепторными вставками; $R_{0}$ и $w$ приведены в $\mathrm{nm}$.

контролирует как медленно, так и быстро затухающие компоненты ФЛ. При повышении температуры процесс переноса энергии усложняется; в частности, он может идти с участием фононов.

\section{2. Эксперимент}

Структуры $\mathrm{Cd}(\mathrm{Zn}) \mathrm{Se} / \mathrm{ZnSe}$ были выращены методом MBE на подложке GaAs. В каждой из них имелось две вставки КТ: донорная, с номинальной толщиной слоя $\mathrm{CdSe} \sim 1.8 \mathrm{ML}$, что соответствовало концентрации $\sim 50 \mathrm{~mol} \% \mathrm{Cd}$ в центре квантовых точек, и акцепторная, с номинальной толщиной слоя $\sim 2.7 \mathrm{ML}$ ( 80-85 mol.\% Cd). Толщина барьера между вставками, $w$, менялась от $8 \mathrm{~nm}$ до $18 \mathrm{~nm}$. Образцы, помимо измерения ФЛ при постоянной накачке, были исследованы методом спектроскопии ФЛ с временным разрешением. Исследование при $5 \mathrm{~K}$ проводились с помощью стриккамеры Hamamatsu, с временным разрешением $\sim 15$ ps. Измерения в диапазоне $10-300 \mathrm{~K}$ проводились методом коррелированного счета фотонов с использованием лавинного однофотонного фотодиода PDM-100-C0E (Micro Photon Devices) и электронного TCSPC модуля SPC-130 (Becker \& Hickl). Для накачки использовалась вторая гармоника Ті-сапфирового фемтосекундного лазера. Лазерный луч, мощностью $2-4 \mathrm{~mW}$, перед окном криостата фокусировался в пятно диаметром 0.5-1 mm на образце.

\section{3. Результаты и обсуждение}

Измерения при низкой температуре (5K) ФЛ из различных образцов показали характерное для FRET ускорение скорости рекомбинации в донорной вставке с уменьшением ширины барьера. Моделирование кривых затухания было проведено, используя уравнение

$$
I(t)=A_{1}\left(\exp \left(-t / t_{1}\right)-\exp \left(-t / t_{r}\right)\right)+A_{2} \exp \left(-t / t_{2}\right) .
$$

Здесь индексы 1 и 2 относятся к быстро и медленно затухающим компонентам ФЛ, соответственно; $A_{1}, A_{2}-$ амплитуды компонент, $t_{1}, t_{2}$ - времена затухания, $t_{r}$ время нарастания после лазерного импульса. Времена затухания донорных КТ при наличии FRET описываются выражением

$$
\frac{\tau_{D}^{0}}{\tau_{D}}=1+\left(\frac{R_{0}}{w}\right)^{6},
$$

где $\tau_{D}^{0}$ - время жизни возбуждения в донорной КТ в отсутствии акцептора (принятая равной величинам для быстрой и медленной компонент в структуре с максимальной толщиной барьера), $\tau_{D}$ принималось равной, соответственно, $t_{1}$ или $t_{2}, R_{0}$ - ферстеровский радиус расстояние, при котором вероятность переноса равна 0.5. На рис. $1, b$ приведены зависимости для медленно и быстро затухающих компонент излучения от расстояния между вставками, построенные так, чтобы выявить линейную зависимость между $\left(w^{-6}\right)$ и $\left(\tau_{D}^{0} / \tau_{D}-1\right) / R_{0}^{6}$. Найдено, что экспериментальные точки ложатся на одну прямую при разных ферстеровских радиусах для быстро и медленно затухающих компонент излучения: $10.5 \mathrm{~nm}$ и $8 \mathrm{~nm}$, соответственно. (Наблюдаемый разброс точек связан, вероятно, с отклонением от номинальных значений толщин барьеров при росте гетероструктур методом МПЭ без вращения подложки.) Меньшая величина $R_{0}$ для медленно затухающих компонент соответствует менее эффективному FRET, что требует более близкого расположения КТ для той же вероятности переноса энергии. К примеру, это могут быть переносы вида 

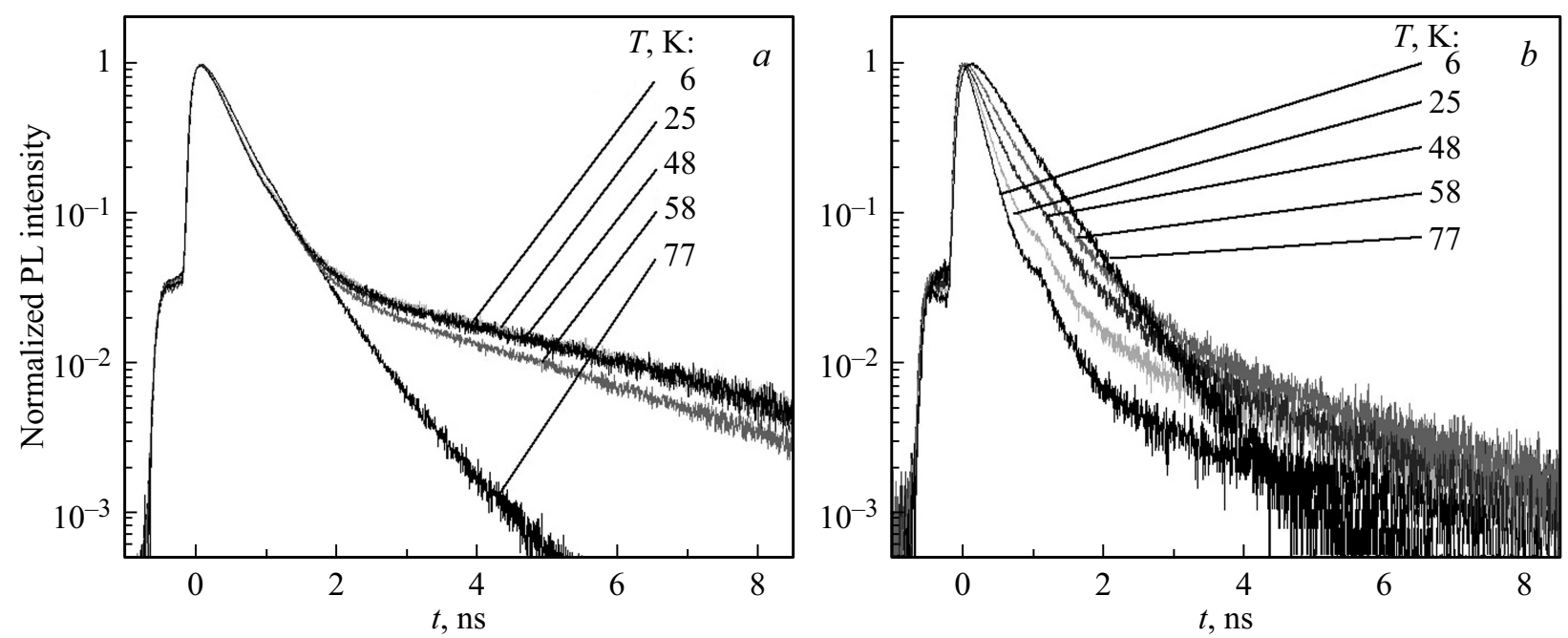

Рис. 2. Кривые затухания ФЛ в структуре с шириной барьера $8 \mathrm{~nm}$, зарегистрированные из пиков: $(a)$ нижнеэнергетической полосы $(b)$ верхнеэнергетической полосы при различных температурах. Длины волн при низкой температуре равны 507 и $478 \mathrm{~nm}$, соответственно.
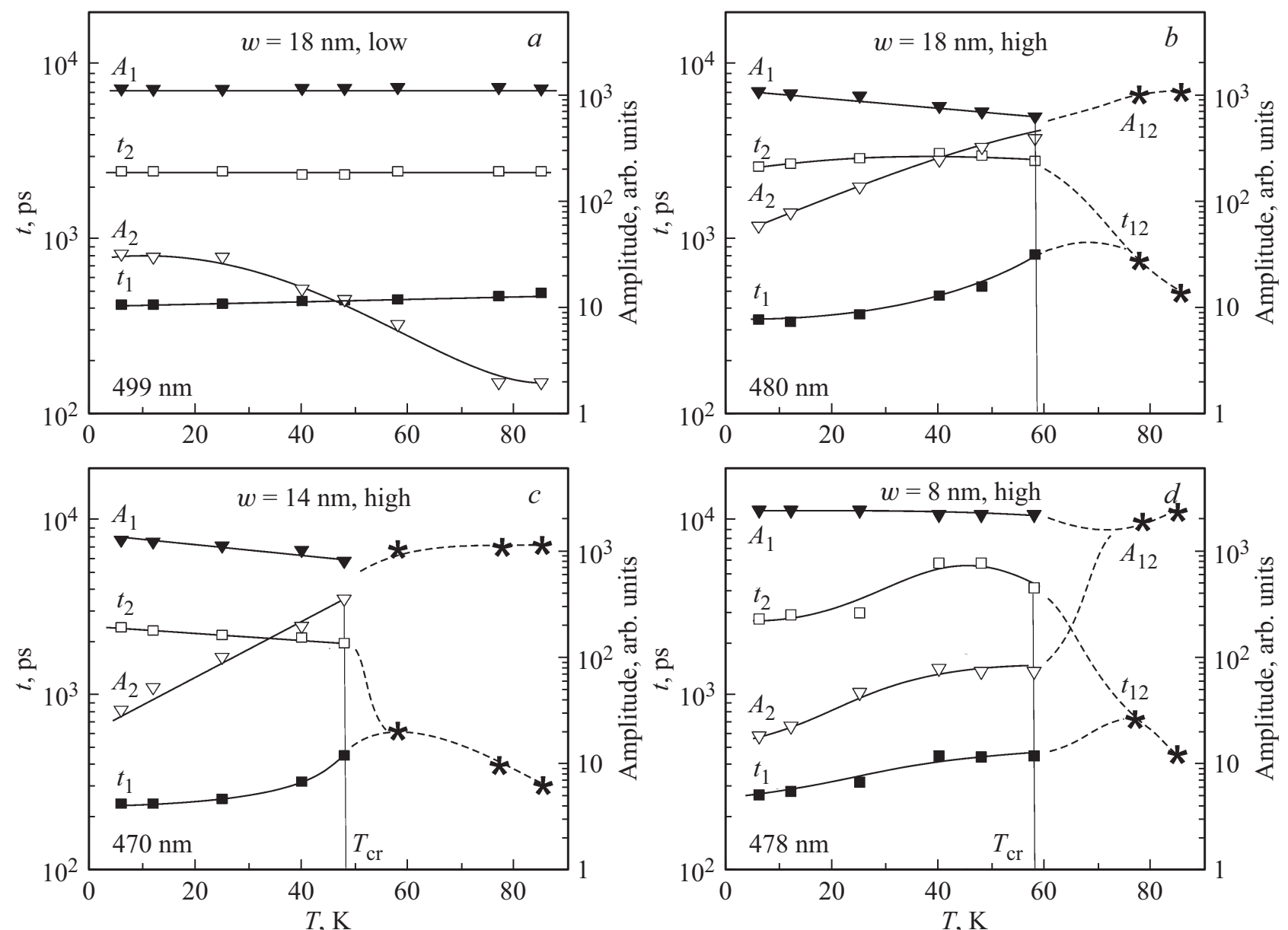

Рис. 3. Характерные времена затухания $t_{1}$ и $t_{2}$ и амплитуды $A_{1}$ и $A_{2}$ быстро и медленно затухающих компонент ФЛ, полученные в результате моделирования кривых затухания, измеренных при различных температурах из пиков $(a)$ акцепторной (low) и $(b)$ донорной (high) полосы в образце с толщиной барьера $18 \mathrm{~nm}$, а также донорных полос из образцов с барьерами (c) $14 \mathrm{~nm}$ и $(d) 8 \mathrm{~nm}$. Длины волн излучения из пиков полос ФЛ при низкой температуре (6 K) указаны на графиках. Звездочки обозначают данные, соответствующие обобществленным состояниям с параметрами $t_{12}$ и $A_{12}$. Экспериментальные точки соединены условными линиями, чтобы показать генезис состояний. 
$\mathrm{FX} \rightarrow \mathrm{FX}$ либо $\mathrm{FX} \rightarrow \mathrm{AX}$, при которых задействовано донорное состояние с большим радиационным временем жизни [7].

Для определения вовлеченности темных экситонов в процесс переноса энергии были проведены измерения при разных температурах. Результаты приведены на рис. 2 и 3. Прежде всего отметим, что характер изменения кривых затухания из верхне- и нижне-энергетических полос различен. В акцепторной полосе наблюдается гашение медленной компоненты при сохранении характерного времени спада быстрой компоненты. В донорной полосе происходит усреднение быстрых и медленных времен затухания ФЛ при подъеме температуры (рис. 2). Иначе говоря, возникает обобществленное состояние, обладающее иным характером релаксации, определяемым временем затухания $t_{12}$ и амплитудой $A_{12}$ (рис. 3).

Особенностью полученных зависимостей является то, что время затухания ФЛ обобществленного состояния донорных полос (звездочки на рис. $3, b-d$ ) достаточно близко к теоретическому пределу при пренебрежении конечными скоростями релаксации (вниз) и заброса (вверх) между этими уровнями. А именно, скорость затухания приближается к величине $\frac{1}{2}\left(\frac{1}{t_{1}}+\frac{1}{t_{2}}\right)$, где $t_{1}$ и $t_{2}$ измерены при $5 \mathrm{~K}$. Этот факт служит аргументом в пользу того, что обобществленное состояние образуется вследствие выравнивания населенностей $\mathrm{FX}$ и $\mathrm{AX}$ уровней.

Для донорных полос характерно возникновение обобществленного состояния при критической температуpe $T_{\text {cr }}$, величина которой (в энергетических единицах) лежит в пределах 4-5 meV. Мы полагаем, что эта величина отчасти определяется расщеплением темных и светлых состояний $\Delta_{\mathrm{AF}}$, которое в первом приближении тем больше, чем меньше размер точки, достигая $\sim 4 \mathrm{meV}$ в $\mathrm{CdSe}$ нанокристаллах [9]. Однако точного соответствия с прогнозируемыми величинами $\left(\Delta_{\mathrm{AF}} \sim 2.5 \mathrm{meV}\right)$ и ожидаемым ходом зависимостей при температурах ниже $T_{\text {cr }}$ не наблюдается. Поэтому мы не можем исключить активационный механизм отмечаемых изменений.

При дальнейшем повышении температуры до $80 \mathrm{~K}$ наблюдается спад времени релаксации обобществленного состояния в донорной полосе ФЛ, так что в зависимости формируется пик, который наиболее выражен в структуpax с широкими барьерами (рис. $3, b, c)$. Мы приписываем такое поведение возникновению конкурирующего канала релаксации носителей из верхних состояний с участием фононов. Этот канал не столь эффективен в структурах с узким барьером из-за свойственной им высокой скорости FRET (рис. 3,d), что и определяет количественную разницу между структурами с барьерами различной толщины.

В акцепторных полосах такого выраженного порогового изменения интенсивности ФЛ нет; здесь чаще наблюдается плавный спад с полным гашением при более высокой температуре. Причем, чем шире барьер, тем монотоннее ход зависимостей интенсивности и слабее меняются времена затухания медленной компоненты (характерный пример приведен на рис. 3, $a$ для структуры с максимально широким барьером). В структуре с узким барьером интенсивная медленная компонента ФЛ сохраняется при более высоких температурах. Такое отсутствие быстрого гашения говорит об эффективном снабжении энергией $\mathrm{FX}$ состояний.

При дальнейшем подьеме температуры до $300 \mathrm{~K}$ наблюдается изменение кривых затухания: моноэкспоненциальный характер, соответствующий установлению термического баланса между населенностью темных и светлых экситонных состояний, нарушается. Кривая затухания становится двух- и даже трехкомпонентной при $300 \mathrm{~K}$. Появляются как более быстрая (50-100 ps), так и более медленная (несколько ns) компоненты, что свидетельствует об активации безызлучательных каналов рекомбинации и центров залипания, соответственно, для термически делокализованных носителей.

\section{4. Заключение}

Нами проведено исследование кинетики рекомбинации в структурах с двумя планарными массивами эпитаксиальных КТ, имеющих разный характерный размер точек и разделенных барьерами различной ширины. Наблюдаемая разница в характере гашения верхне- и нижне-энергетических полос ФЛ может быть объяснена эффективным ферстеровским резонансным переносом энергии от донорных к акцепторным КТ. Моноэкспоненциальный характер затухания ФЛ из верхнеэнергетической полосы, достигаемый при температурах $\sim 70 \mathrm{~K}$, соответствует, предположительно, состоянию термического равновесия между темными и светлыми экситонами. Подавление при этом затухания медленной компоненты в акцепторных точках связано с эффективным переносом энергии на состояния не только светлых, но и темных экситонов. При дальнейшем нагреве структуры включаются более сложные механизмы затухания с участием фононов, нерадиационных каналов и центров залипания. Точное определение вклада различных механизмов требует детального моделирования экспериментальных результатов. Полученные данные могут быть полезны для анализа параметров нанофотонных и оптоэлектронных приборов.

\section{Список литературы}

[1] S.V. Ivanov, A.A. Toropov, T.V. Shubina, S.V. Sorokin, A.V. Lebedev, I.V. Sedova, P.S. Kop'ev. J. Appl. Phys. 83, 3168 (1998).

[2] T.V. Shubina, A.V. Rodina, M.A. Semina, A.A. Golovatenko, A.A. Toropov, M.V. Rakhlin, I.V. Sedova, S.V. Sorokin, S.V. Gronin, A.A. Sitnikova, D.I. Kuritsyn, S.M. Sergeev, Z.F. Krasil'nik, S.V. Ivanov. Phys. Status Solidi B 253, 1485 (2016). 
[3] T.V. Shubina, M.A. Semina, K.G. Belyaev, A.V. Rodina, A.A. Toropov, V.S. Ivanov. J. Electron. Mater. 46, 7, 3922-3926 (2016).

[4] T.V. Shubina, G. Pozina, A.A. Toropov. Phys. Status Solidi B 254, 42016.

[5] T. Förster, Ann. Phys. (Leipzig) 437, 55 (1948).

[6] F. Liu, A.V. Rodina, D.R. Yakovlev, A.A. Golovatenko, A. Greilich, E.D. Vakhtin, A. Susha, A.L. Rogach, Y.G. Kusraev, M. Bayer. Phys. Rev. B 92, 125403 (2015).

[7] A.N. Poddubny, A.V. Rodina. ЖЭТФ 149, 614 (2016).

[8] B. Valeur, M. Berberan-Santos, Excitation Energy Transfer, Molecular Fluorescence: Principles and Applications. 2nd ed. Wiley-VCH, Weinheim, (2012). Ch. 8.

[9] O. Labeau, P. Tamarat, B. Lounis. Phys. Rev. Lett. 90, 257404 (2003).

Редактор Д.В. Жуманов 